Review

\title{
Colorectal Cancer Biomarkers and the Potential Role of Cancer Stem Cells
}

\author{
Russell C. Langan ${ }^{1}$, John E. Mullinax ${ }^{2}$, Manish T. Raiji ${ }^{1}$, Trevor Upham ${ }^{1}$, Thomas Summers ${ }^{3}$, Alexander \\ Stojadinovic ${ }^{4,5}$, Itzhak Avital ${ }^{凶}$ \\ 1. Georgetown University Hospital, Washington, DC, USA \\ 2. University of South Florida Medical Center, Tampa, FL, USA \\ 3. Department of Anatomic Pathology, Walter Reed National Military Medical Center, Bethesda, MD, USA \\ 4. Department of Surgery, Division of Surgical Oncology, Walter Reed National Military Medical Center, Bethesda, MD, USA \\ 5. Department of Surgery, Uniformed Services University of the Health Sciences, Bethesda, MD, USA \\ 6. Bon Secours Cancer Institute, Richmond, VA, USA.
}

Corresponding author: Itzhak Avital, MD, FACS. Medical Director, Bon Secours Cancer Institute, 5855 Bremo Road Suite 506, Richmond, VA 23226. Phone: (804) 221-7364 Itzhak.Avital@gmail.com.

(c) Ivyspring International Publisher. This is an open-access article distributed under the terms of the Creative Commons License (http://creativecommons.org/ licenses/by-nc-nd/3.0/). Reproduction is permitted for personal, noncommercial use, provided that the article is in whole, unmodified, and properly cited.

Received: 2013.01.05; Accepted: 2013.02.1I; Published: 2013.03.15

\begin{abstract}
Over $50 \%$ of patients with colorectal cancer (CRC) will progress and/or develop metastases. Biomarkers capable of predicting progression, risk stratification and therapeutic benefit are needed. Cancer stem cells are thought to be responsible for tumor initiation, dissemination and treatment failure. Therefore, we hypothesized that CRC stem cell markers (CRCSC) can identify a group of patients whom are at increased risk for recurrence or progression of disease. If proven correct, these CRCSC biomarkers may herald a paradigm shift in the treatment of this deadly disease. This manuscript reviews current CRC evidence based screening modalities, patient stratification, and summarizes the current state of biomarkers and discusses the novel concept of putative CRCSC's as prognostic biomarkers.
\end{abstract}

Key words: Colorectal cancer, Cancer stem cells, Biomarkers.

\section{Introduction}

Colorectal cancer (CRC) is the third most common cause of cancer-related death in the US ${ }^{1}$. One out of four patients presenting with conventionally staged node negative disease (AJCC Stage I and II), and over $50 \%$ of patients with Stage III disease will develop local recurrence and/ or metastases 2,3 . Once metastases become clinically evident, prognosis is extremely poor and survival is often measured in months. Moreover, in spite of the fact that modern systemic therapies for CRC have resulted in improved overall survival (OS), failure rate in the adjuvant setting is $30 \%$ for high-risk Stage II and Stage III patients, and overall response rate is only $60 \%$ for patients with Stage IV CRC 4,5,6. A significant advance in the care of these patients could be realized by biomarkers that can accurately identify patients at-risk for disease recurrence and dissemination, along with those that fail to respond to systemic therapy. These patients might benefit from early aggressive treatment, alternative treatment strategies, and/or frequent surveillance for and early detection of disease recurrence.

Until more effective therapies can be developed, one strategy for improving outcomes while reducing the socio-economic burden of CRC is to develop novel strategies for CRC screening that will result in higher rates of early CRC detection. Another strategy to improve outcomes in this patient-centered, quality-driven, and value-based purchasing era in oncol- 
ogy is to identify those patients who would most benefit from adjuvant treatments and intensive post-treatment surveillance protocols. There is an acute need for highly sensitive and specific biomarkers capable of identifying patients with early CRC that are highly likely to recur and/ or metastasize, and patients who are likely to progress despite adjuvant therapy. Such risk stratification would: [1] spare low risk patients likely cured by surgery alone the toxicity of systemic therapy; [2] identify and treat at-risk patients with early stage CRC; and, [3] limit treatments to patients with clinically latent or stable residual disease, thereby reserving additional therapy for treatment-responsive disease progression ${ }^{7}$. The aim of this document is to address current CRC evidence based screening modalities and patient stratification, summarize the current state of biomarkers and discuss the novel concept of putative CRCSC's as prognostic biomarkers.

\section{Current State of Screening Methods}

The sequence of progression from adenoma to invasive carcinoma in CRC, along with the accessibility of the lower gastrointestinal tract to endoscopic imaging has led to the development of screening protocols based around direct imaging of colonic mucosa $^{8}$. Currently, the United States Preventive Services Task Force (USPSTF) recommends CRC screening that involves a combination of stool-based laboratory studies and direct visualization of colonic mucosa 9. The gold standard for CRC screening is currently colonoscopy, beginning at the age of 50 in those without family history of CRC. In a case controlled comparison of patients undergoing colonoscopy screening with historical patients who did not undergo screening, the use of colonoscopy reduced mortality risk, with an odds ratio of $0.41^{10}$. An alternative screening modality is sigmoidoscopy every 5 years combined with high-sensitivity fecal occult blood testing (FOBT) every three years. The introduction of immunochemical-FOBT has increased the sensitivity of stool-based screening in detecting advanced adenomas and early CRC ${ }^{11}$. Lastly, annual FOBT is an option endorsed by the USPSTF.

Given the invasive nature of direct colonic visualization, indirect methods of evaluating the colonic mucosa have been developed. Double-contrast barium enema (DCBE) involves radiographic imaging of the colon following the instillation of barium followed by distension of the colon with air. This modality is associated with unacceptably high false negative rates, especially for polyps less than $1 \mathrm{~cm}$ in size, to be recommended as a valid screening evaluation ${ }^{12}$. Computed tomography colonoscopy involves thin-slice reconstruction of the colon for radiographic evaluation of the mucosa. While this evaluation may be more desirable to patients, a large meta-analysis showed that either of these studies were less sensitive for small polyps than colonoscopy ${ }^{13,14}$. Capsule endoscopy has been evaluated as another possible indirect method of visualizing the colonic mucosa, but it also lacks adequate sensitivity to be used as a screening test ${ }^{15}$.

The isolation of stool DNA, derived from sloughed mucosal and CRC cells, and the subsequent identification of CRC-associated genetic mutations in stool samples has been described ${ }^{16}$. DNA amplification techniques have improved the nucleic acid yield in stool samples ${ }^{17}$, opening the possibility of screening for specific genetic mutations associated with CRC. A commercial test has been developed, which detects k-ras, APC, and p53 gene mutations, along with long DNA and the microsatellite instability marker BAT-26. A blinded comparison of the findings of this DNA based test showed a sensitivity for advanced adenomas and invasive carcinoma of only $51 \% 18$

Current problems with screening tests for CRC include the lack adequate sensitivity, low cost-effectiveness, general invasiveness, and poor compliance. The current gold standard of colonoscopy demonstrates significant variability between providers in adenoma detection during colonoscopy 19 . The rate of CRC screening using current guidelines is quite low, with only $5 \%$ to $60 \%$ of insured patients achieving adequate screening 2021 , with even lower rates in uninsured and minority patient populations ${ }^{22}$. This has led to the development of serum-based tests that would be significantly less invasive and more universally available with the hope of increasing compliance.

The most often studied serum tumor marker is carcinoembryonic antigen (CEA), which has been widely used in the surveillance of patients following primary surgical resection of CRC ${ }^{23}$. Carbohydrate antigen 19-9 (CA19-9) is another heavily studied serum marker of CRC as well as pancreatic carcinoma, and may have prognostic value in patients with diagnosed CRC ${ }^{24}$. When considering plasma CEA levels in patients with in situ carcinoma excised at colonoscopy (T0 polyps), the sensitivity is even lower ${ }^{25}$. Along with CEA and CA19-9, over 50 different serum markers have been evaluated as screening modalities for CRC, but a large meta-analysis has shown that all of these have low sensitivities when evaluated alone ${ }^{26}$. One such investigation of circulating DNA markers for K-ras mutations found only 22 of 62 patients with adenomas and 5 of 8 patients with carcinoma had 
detectable K-ras mutations in the serum ${ }^{27}$.

These initial evaluations of serum markers for CRC screening demonstrate low sensitivity and specificity, especially when considering early adenomas. Current experimental CRC screening tests also have unacceptably low sensitivity and specificity profiles, and are less efficacious for adenomas than for advanced carcinomas, making them ineffective for cancer screening. Additionally as highlighted above, even the best current screening methods are flawed in both delivery and compliance, illustrating the need for novel screening approaches.

\section{Biomarkers for Screening and Follow-up for Cancer: Positives and Pitfalls}

The National Institute of Health (NIH) defines a biological marker (biomarker) as a biological molecule found in blood, other body fluids, or tissues that is a sign of a normal or abnormal process, or of a condition or disease ${ }^{28}$. In addition to screening for disease, a biomarker may be used to evaluate how well the body responds to a treatment and can also be called a molecular marker or signature molecule ${ }^{28}$. A biomarker can exist as related to DNA, RNA, micro-RNA, epigenetic changes, protein and even antibody expression. Biomarker discovery is increasing with the modern throughput of medical research in genomic and proteonomics. New biomarkers are building upon growing information at the same time that research tools are becoming cheaper and more efficient. Currently, biomarkers are routinely used to help guide treatment decisions in lung cancer ${ }^{28}$, breast cancer $^{29}$, and colon cancer ${ }^{30}$ with simple testing of existing tumor samples.

Though many biomarkers are interesting in their cell function as related to malignancy, biomarkers such as telomerase, transforming growth factors (TGFa and TGF $\beta$ ), epidermal growth factors (erbB2 and erbB3) and mucin (MUC1 and MUC2) have not shown clinical utility ${ }^{31}$. Prostate specific antigen (PSA), carcinoembrionic antigen (CEA) and cancer antibody maker 125 (CA-125) have proven observational associations with malignancy but lack specificity. Moreover, a reduction in mortality has not been shown using these biomarkers ${ }^{32}$. Fecal occult blood test (FOBT) is the only known protein biomarker shown to decrease cause-specific mortality in cancer screening 33 .

Biomarkers have the potential to change treatment and diagnostic algorithms across a broad spectrum of patients. Risk stratification for screening might be augmented by finding biochemical signatures that recognize either a predisposition to disease alone or as a complement to existing tests. For exam- ple, particular HLA alleles in conjunction with certain human papillomaviruses can increase the risk of cervical cancer and could change the screening algorithm ${ }^{34}$. Tissue biomarkers are at the forefront of discovery and investigations are ongoing regarding their prognostic value and the ability to accurately guide therapy.

\section{Prognostic Colorectal Cancer Biomarkers}

Extensive CRC research over the last decade has suggested promising biomarkers ${ }^{35}$. Although many biomarkers have been described, only a select few have provided prognostic data. This list includes markers such as epidermal growth factor receptor (EGFR), BRAF, tumor MSI-H expression (defects in DNA mismatch repair, MSI phenotype), 18q AI expression, p53 expression and KRAS mutation. Decisions regarding the modality and combination of treatments for CRC patients are made based on a variety of clinical factors in an effort to deliver patient-specific care proven to be efficacious without untoward adverse effects. This set of clinical factors has been revised and expanded as radiographic and laboratory techniques have improved. The physician today must decide eligibility for specific treatment based on an evolving set of diagnostic tests based on molecular profile of the patient's tumor. This profile is based on a number of mutations common to many patients which, when correlated with survival data, provide meaningful criteria for treatment decision making.

Therapy targeting the epidermal growth factor receptor (EGFR) has been studied extensively for patients with metastatic $\mathrm{CRC}^{36,37}$. In the largest experience, the addition of cetuximab to standard cytotoxic chemotherapy conferred a progression-free survival benefit but no benefit in overall survival. On further analysis, this benefit was only found in the Kirsten-RAS (KRAS) wild-type patients. This is because a mutation in the KRAS gene leads to constitutive activation of the EGFR signaling pathway, thereby abrogating any effects of upstream EGFR blockade. This finding has led to the selection of patients for EGFR blockade therapy based on KRAS status, with cetuximab only offered to those patients with no KRAS mutation in the primary tumor ${ }^{37-39}$.

In a similar fashion, a small percentage of primary CRC contains a mutation in the BRAF gene. The BRAF(V600E) mutation in CRC confers poor survival with standard chemotherapy regimens ${ }^{37,38}$. Vemurafenib, a small molecule inhibitor of the mutant protein, was recently approved for use in patients with metastatic melanoma harboring the BRAF(V600E) mutation. Interestingly, CRC patients do not enjoy 
response rates similar to patients with $\mathrm{BRAF}(\mathrm{V} 600 \mathrm{E})$ melanoma when given vemurafenib. The reason for this therapeutic failure was recently shown to be caused by compensatory increased signaling upstream at the level of the EGFR. By adding cetuximab for EGFR inhibition to a standard regimen of vemurafenib, a significant antitumor response was shown in vitro and in vivo ${ }^{40}$. While there are no reported results of this combination therapy in adult patients, the laboratory results are promising and may offer another option for late stage CRC patients with wild-type KRAS and the BRAF(V600E) mutation.

While CRC may develop through a sequence of acquired mutations, another theory of carcinogenesis implies that microsatellite instability (MSI) may play a role. ${ }^{41}$ When mutations occur in mismatch repair (MMR) genes such as MSH2, MSH6 and MLH1 the tumor is referred to as mismatch repair deficient (dMMR). With the ability to repair single nucleotide polymorphisms lost, instability develops in microsatellites - sites in the genome normally comprised of dinucleotide repeats. ${ }^{41}$ Individual tumors can be assayed for the level of MSI and are described as MSI-High, MSI-Low, and microsatellite stable (MSS). ${ }^{42}$ MSI-H phenotype has been associated with improved clinical outcome (disease-free and overall survival) ${ }^{43,44}$. In addition, some published data supports the recommendation not to administer chemotherapy to Stage II patients with a MSI-H primary colon cancer ${ }^{45}$. The overall predictive value of MSI-H phenotype is currently being tested in the Eastern Cooperative Oncology Group Trial 5202 (E5202) adjuvant CRC trial ${ }^{35}$. This trial is specifically intended to identify patients with AJCC Stage II CRC most likely to respond to adjuvant systemic therapy.

Other mutations can yield prognostic information. Tumor expression of 18q AI has been associated with decreased survival and is currently being studied in the E5202 trial ${ }^{35}$. A mutation in the tumor suppressor gene p53 has been associated with poor prognosis, including decreased disease free survival (DFS), recurrence free survival (RFS) and overall survival $(\mathrm{OS})^{35}$.

These validated criteria are changing the way patients are treated and also how the response to treatment is judged. Using modern molecular biology methods, findings from the laboratory are being translated to the clinic in an effort to focus therapy on patients who are most likely to benefit. As therapies become more sophisticated, the methods of patient selection must follow. In order to derive the most efficacious results from therapies with not insignificant side effects, these methods must be employed and others must be developed.

\section{Putative Colorectal Cancer Stem Cell-Associated Biomarkers}

The methods of patient stratification based on tumor mutation status have resulted in significant advances for patients with CRC; however, in order to expand upon these advances new criteria must be developed to further stratify patients. The precise cell of origin of CRC remains unknown. Recently, compelling evidence has emerged in support of the cancer stem cell (CSC) hypothesis in several solid organ epithelial malignancies including $\mathrm{CRC}^{46,47,48-51}$. The theory of the cancer stem cell was originally proposed by Cohnheim in $1875{ }^{52}$.This theory encompassed four principles: 1. A number of external or internal insults, such as radiation, injury, or carcinogens, may result in genetic damages in the stem cells; 2. Each damaged stem cell gives rise to a morphologically distinct type of tumor; 3 . All the cells within a given tumor share the same profile at different stages of progression; 4 . Different tumors from different stem cells have different genetic and biochemical profiles ${ }^{52}$. Following this, the theory of clonal evolution was introduced by Nowell in 1976 stating, 1. A number of external or internal stem cell insults, such as radiation, injury,or carcinogens, may result in genetic damages in stem cells; 2 . A genetically damaged stem cell gives rise to a morphological distinct type of tumor; 3 . New cell clones constantly emerge from the same tumor; 4 . Only the more aggressive ones with growth advantages progress, and from which, new clones emerge 53 . Currently, both of which have been supported by several lines of evidence ${ }^{54}$.

The CSC hypothesis posits that CSC's are responsible for tumor initiation, metastases and resistance to treatment leading to disease relapse following surgery and/or chemoradiotherapy ${ }^{55}$. The traditional, stochastic model of tumorigenesis suggests that all cells within a tumor are capable of tumor initiation and propagation ${ }^{56}$. The CSC hypothesis proposes a hierarchical model, in which only a small fraction of cells (CSC) are capable of tumor propagation $^{56}$. The CSC hypothesis therefore raises questions regarding current diagnostic and therapeutic modalities, suggesting that the CSC is a rational target for the development of more efficacious screening, early detection, prevention, treatment and surveillance modalities and interventions ${ }^{56,57}$. Based on the tenets of the CSC hypothesis, identification, proper selection, characterization, testing, biological implications and validation of CRC derived CSC (CRCSC) are imperative for improving early detection, screening, risk stratification prognostication and individualized prediction of treatment response. 
Properties that define potential $\mathrm{CSC}^{\prime}$ s are: [1] self-renewal; [2] the capacity for differentiation (allowing for recapitulation of all cell types of the original tumor); [3] tumor initiating capacity; and, [4] asymmetric cell division via non-random chromosomal co-segregation ${ }^{49,58}$. Investigators have used these properties and various membrane and cytoplasmic markers to isolate putative CRCSC: CD133, CD24, CD29, CD44, CD166 (ALCAM), EpCAM, Lgr5, ALDH1A1 and ALDH1B1 (Table 1). These markers represent all reported CRCSC. Despite the potential of CRCSC's to be utilized as clinically relevant biomarkers, little is known about the prognostic value of non-CD133 CRCSC markers ${ }^{59}$. Notwithstanding, the CSC hypothesis may herald a paradigm shift in screening and early detection in CRC once the precise role of CRCSC markers is further established. Therefore, we hypothesize that CRCSC markers can be used as biomarkers to predict disease progression, and identify patients at risk for recurrence.

Two fundamental issues regarding colorectal carcinogenesis remain unanswered. First, the level of differentiation in the initiating neoplastic cell has not been well described i.e. colonic stem cell vs. differentiated mature colonic mucosal epithelial cell. Second, since tumors are well known to be composed of a heterogeneous group of cells, the specific identity of tumor cells that lead to lymph-node involvement, and metastatic disease is not well characterized. ${ }^{56}$ Recently, attempts to address both of these issues pragmatically, which are critical to our understanding of tumor biology, have resulted in the description of cancer stem cells. While the stochastic model of tumorigenesis holds that every cell within the tumor population is capable of tumor initiation and propagation, the cancer stem cell model proposes that only a small fraction of cells possess the ability to initiate cancer growth and promote metastatic dissemination ${ }^{56}$. We posit that only stem cells possess the potential for unlimited proliferation, multi-lineage differentiation, and colonization at new sites, and thus, represent the most likely precursor for invasive and metastatic CRC. Therefore, these cells require much further attention and introspection.

Table I. Summary of putative CRC Stem Cell Markers.

\begin{tabular}{|c|c|c|}
\hline Marker & Gene & Function \\
\hline CD133 & PROML1 & $\begin{array}{l}\text { * Transmembrane glycoprotein } \\
\text { * Associated with primitive cells } \\
\text { * Initially found that CD133 positive colon tumor cells were 200-fold enriched for CSCs compared to unsorted } \\
\text { tumor cells and that colon tumors contained a higher percentage of CD133 positive cells than normal tissue; now } \\
\text { controversial }\end{array}$ \\
\hline CD24 & $C D 24$ & $\begin{array}{l}\text { * Cell adhesion molecule } \\
\text { * A ligand of p-selectin on tumor cells that is associated with in vitro invasiveness } \\
\text { * Strong expression is a poor prognostic factor in CRC }\end{array}$ \\
\hline CD29 & ITGB1 & $\begin{array}{l}\text { * Integrin that mediates cell-ECM adhesion and is involved in homing to sites of inflammation } \\
\text { * Involved in cell growth, differentiation, migration and death }\end{array}$ \\
\hline CD44 & $C D 44$ & $\begin{array}{l}\text { * Cell surface glycoprotein involved in cell adhesion and migration } \\
\text { * Associated with malignant progression (adenoma to carcinoma): involved in cell migration through the ECM } \\
\text { * Enhanced expression in breast cancer epithelial cells facilitated tumor cell migration }\end{array}$ \\
\hline CD166 & $A L C A M$ & $\begin{array}{l}\text { * Involved in neuronal extension, embryonic hemopoiesis, embryonic angiogenesis } \\
\text { * Cell adhesion molecule } \\
\text { * Associated with adenoma to carcinoma development }\end{array}$ \\
\hline EpCAM & EPCAM & $\begin{array}{l}\text { * Cell adhesion molecule } \\
\text { * Linked to Cadherin-Cathenin pathway and Wnt pathway } \\
\text { * Expression data linked to poorer survival times in several tumor types including breast cancer } \\
{ }^{*} \text { Loss of expression associated with aggressive rectal cancer }\end{array}$ \\
\hline ALDH1A1 & $A L D H 1 A 1$ & $\begin{array}{l}\text { * Detoxifying enzyme responsible for the oxidation of intracellular aldehydes } \\
\text { * Early differentiation of stem cells } \\
\text { * Involved in resistance to chemotherapy (alkylating agents) } \\
\text { * Malignant prostate stem cells and predictor of prostate cancer patient outcome }\end{array}$ \\
\hline ALDH1B1 & ALDH1B1 & $\begin{array}{l}\text { * Detoxifying enzyme responsible for the oxidation of intracellular aldehydes } \\
\text { * Early differentiation of stem cells } \\
\text { * Higher expression in CRC }\end{array}$ \\
\hline Lgr5 & LGR5 & $\begin{array}{l}\text { * Associated with intestinal stem cells } \\
\text { * Downstream target of Wnt pathway }\end{array}$ \\
\hline
\end{tabular}

Abbreviations: CD, cluster of differentiation; ALDH, aldehyde dehydrogenase-1; ECM, extra cellular matrix; CRC, colorectal cancer; Lgr, leucine-rich-repeat-containing G-protein. 
There are various methods of CSC identification in vitro; however, there is currently no consensus on a universally acceptable method ${ }^{48-51,56,59}$. For technical reasons, the most commonly utilized method of identification involves separating cells based on proteins thought to be associated with the cancer stem cell phenotype ${ }^{60}$. Detection of sub-populations of cells such as CRCSC in regional nodes negative for tumor cells by conventional histopathology, or as circulating cells in the blood or bone marrow, is likely to increase accuracy of both cancer staging and prognosis. While some studies have linked CSC markers to prognosis, there remains no definitive association between putative CSC markers and disease behavior, disease progression, or survival. An association between putative CRCSC markers and survival could not only improve cancer screening and early detection, but also help define optimal post-treatment follow up. Equally as important, CRCSC markers could potentially tailor more efficacious treatment modalities to those patients who stand to benefit most, such as at-risk node-negative (early stage) CRC patients who are prone to recur.

Molecular biomarkers provide potential benefits because they enable identification of specific cell types and cell populations that are associated with disease behavior and clinical outcomes ${ }^{60}$. Methods of cancer stem cell identification are based on markers specific for normal progenitor or stem cells in the same organ ${ }^{61}$. The adult stem cells of the colon are of particular interest because they sustain the perpetual self-renewal of healthy colonic epithelium and are therefore able to acquire the number of mutations required for carcinogenesis ${ }^{60}$. Although initial reports identified CD133 as a reliable CSC marker in CRC, subsequent studies have shown that CD133 expression is not restricted to rare cell subsets and it is detectable in a large majority of tumor cells, irrespective of their tumorigenicity ${ }^{59}$. Lugli et al also stated that neither over-expression nor loss of CD133 was significantly associated with tumor progression or survival ${ }^{59}$. In fact, several manufactures have stopped producing antibodies for CD133. Alternatively, CD29, C44, EpCAM, CD166, ALDH1A1 and ALDH1B1 were reported to identify another CSC pool ${ }^{59,62}$. Presence of these proteins has been associated with characteristics of stemness both in vitro and in vivo ${ }^{56}$. Cells positive for these markers have been shown to generate tumors recapitulating the primary tumor with increased clonogenic ability and multi-lineage potential ${ }^{56}$. This subset of cells has also been associated with tumor stage, differentiation, invasiveness, metastasis formation, and prognosis ${ }^{56}$. However, there is a paucity of longitudinal data in order to more fully understand the related disease biology, as is presented in our study.

\section{CD29}

Uncertainty remains as to whether CSC markers exclusively delineate the stem cell population and whether they can predict disease behavior 4,60 . In normal colon, CD29 has been observed in the lower parts of the crypts and therefore has been hypothesized to be a mucosal progenitor cell marker63. The combination of CD24 and CD29 has been suggested to identify the tumor initiating fraction in mouse colon carcinomas $^{63}$ and it is proposed that CD29 may promote cancer progression by inducing invasion, migration and metastasis through regulation of the tumor microenvironment ${ }^{63,64}$. Zou et al also stated that the CD133+CD29+ cellular fraction up-regulated self-renewal, proliferation and differentiation, and therefore reported CD29 to be a new stem cell marker for colon cancer, ${ }^{64}$ although its full biological function has yet to be elucidated. In addition, our lab has identified a possible association between CD29 expression and overall survival in CRC. Synonymous to the published data above, we found a statistical significance with worse overall survival to decreased CD29 expression $(p=0,052)$ when assessing thirty CRC patients ${ }^{65}$. Specifically, loss of CD29 exhibited a trend toward association with survival $(p=0.098)$ after adjusting for the effect of stage and CD29 expression was higher in normal colonic tissue $(p=0.014) 65$. Also, lymph node metastases were associated with a trend toward decreased CD29 expression 65

\section{CD44}

CD44 has long been thought to be a marker of tumor invasiveness and metastasis, and more recently has been described as a potential CRCSC marker, although controversial ${ }^{57,59}$. CD44 is a transmembrane glycoprotein that can act as a receptor for extracellular matrices such as hyaluronic acid; it is a downstream target of the Wnt/B-catenin pathway ${ }^{57}$. High expression of CD44 in malignant colonic epithelial cells has been associated with tumor virulence; knockdown of CD44 in primary colon cancer cell lines reduced clonogenicity in vitro and tumorigenicity in vivo ${ }^{63}$. Currently, it is not known whether CD44 is a specific marker for stem cells, as a large population of CD44-exressing cells within a tumor has many splice variants ${ }^{57}$. Early reports of the CD44 gene and its splice variants describe decreased overall survival in patients with increased expression levels of the gene or its related protein. More recent results, however, are inconsistent; some suggest either no role for CD44, others demonstrate a worse clinical outcome with loss 
of protein expression $66,67,68,59$. Further, some have identified increased expression of CD44 in colon along the progression from normal colonic epithelium to adenoma to carcinoma ${ }^{68,59}$. We did not find CD44 to be prognostic with relation to survival, grade of tumor, lymph node metastases or changes in normal to primary or primary to metastatic tissue 65 .

\section{EpCAM}

Epithelial cell adhesion molecule (EpCAM) is a transmembrane glycoprotein mediating epithelium-specific intercellular adhesion. It is also a molecule involved in cell signaling, migration, proliferation and differentiation ${ }^{69}$. As EpCAM is expressed wholly in epithelium-derived cancers, it has been reported as a diagnostic marker ${ }^{69}$. Enhanced EpCAM expression has been linked to advanced cancer stage and worse overall survival ${ }^{69}$, specifically for breast cancer and gallbladder cancer ${ }^{70,71}$. EpCAM expression has also been linked to higher primary tumor grade and inferior local and distant recurrence free survival in rectal cancer ${ }^{72}$. Lugli et al. also noted EpCAM to be a prognostic marker; however, they found a decreased expression of EpCAM to be prognostic. In addition, decreased EpCAM expression was found to be significantly associated with infiltrating tumor margin, tumor invasion, and presence of lymph node metastasis ${ }^{59}$. Similarly, we found that decreased EpCAM expression is associated with increasing lymph node stage $(\mathrm{p}=0.06)\left(\mathrm{N} 0\right.$ vs. $\mathrm{N} 1 \mathrm{vs}$. N2) ${ }^{65}$.

\section{CDI66}

CD166 expression can be used to further select CSC's within the EpCAM(+)CD44(+) population ${ }^{57}$. There appears to be heterogeneous expression of CD166 in CRC and the expression level is associated with poor clinical outcomes. It has been hypothesized that this effect is possibly mediated by CD166 regulation of cell-to-cell interactions ${ }^{63}$. In immunodeficient mice, CD44+CD166+ colon cancer cells display high tumor initiating potential ${ }^{63}$. Patel et al. found a significant increase in CD166 expression in adenomatous glands and an age-dependent increase in CD44 and CD166 expression. This was also associated with the number of colon polyps ${ }^{59,73}$. Weichert et al. described increased expression of CD166 in tumor tissue, and, in a group of 111 CRC cases, CD166 expression correlated with shortened overall survival ${ }^{59,74}$. These findings suggest a role for both CD44 and CD166 in the colon adenoma to carcinoma sequence. It is possible that because of the small number of patients analyzed in this study an association between CD44, CD166 and outcomes could not be elucidated. We did not find CD166 to be prognostic with relation to survival, grade of tumor, lymph node metastases or changes in normal to primary or primary to metastatic tissue 65 .

\section{CD24}

CD24 has also been implicated in CRCSC's. Choi et al examined 523 colorectal adenocarcinomas of various stages and found significant correlation between CD24 expression and degree of differentiation 55,75 . Other studies have shown that the degree of colorectal tumor CD24 expression is associated with lymph node metastasis and shortened patient survival $55,76,77$. We chose not to use CD24 in our study. Although there may be a relationship between CD24 and oncologic outcome, the evidence for CD24 as CRCSC marker is less convincing.

\section{ALDH I}

ALDH1 has been reported as a cancer stem cell marker in pancreatic, breast, prostate, and lung cancer, multiple myeloma and leukemia. More recently ALDH1 was identified as a CRCSC marker ${ }^{57}$. ALDH1 functions by catalyzing the irreversible oxidation of a range of aliphatic and aromatic aldehydes to their corresponding carboxylic acids ${ }^{6}$. High ALDH1 activity is detected in stem and progenitor cells of various lineages. Enhanced ALDH1 expression was found in areas where epithelial progenitor cells localize within normal breast, colon and stomach ${ }^{61}$. Various groups have reported that high ALDH1 expression is associated with reduced survival times in breast, lung, pancreas, bladder and prostate cancer patients ${ }^{61}$. Deng et al. found that ovarian cancer patients with high ALDH1 had shorter disease free and overall survival compared to those with low ALDH1 ( $p=$ 0.0036 and $p=0.0023$, respectively $)^{61}$. Lugli et al. also noted that increased ALDH1 expression is associated with higher tumor grade ${ }^{59}$. We similarly found an association between ALDH expression and disease behavior ${ }^{65}$. Greater expression of ALDH1A1 was associated with increasing stage $(\mathrm{p}=0.042$ over stages 2 , $3 b, 3 c$, and 4) ${ }^{65}$. Compared to normal colon tissue, primary tumors were associated with increased expression of ALDH1B1 $(p=0.008){ }^{65}$. ALD1H1B1 expression level differed according to whether the tumor was moderately or poorly differentiated, well differentiated, or mucinous; the highest expression levels were associated with moderately or poorly differentiated tumors $(p=0.011){ }^{65}$. Compared to normal colon tissue metastatic colon cancers from different patients were associated with increased ALDH1B1 expression $(\mathrm{p}=0.001){ }^{65}$.

\section{LGR5}

Investigators studying the origin of normal co- 
lon progenitor cells found exclusive expression of leucine-rich-repeat-containing G-protein-coupled receptor 5 (Lgr5) in the crypt cells of the colon. The Lgr5 gene is a downstream target of the Wnt pathway and cells expressing this receptor were able to recapitulate all epithelial lineages in just 60 days. ${ }^{78}$ Subsequently it was shown that Lgr5 expression may be required for maintenance of stem cell niche since loss of expression leads to invasion and upregulation of genes associated with epithelial to mesenchymal transition (EMT). ${ }^{79}$ In a recent publication, Merlos-Suarez et al stated that, patients bearing primary CRCs with high average expression of intestinal stem cell genes including Lgr5, had a relative risk of relapse 10-fold higher than those with low levels $(p<0.0001){ }^{80}$. Finally, a Japanese report indicated that high Lgr5 expression is correlated with a decreased disease free survival and a higher rate of hepatic and lymph node metastasis ${ }^{81}$. Based on these findings, the expression of Lgr5 should be prospectively studied and correlated with response to treatment in an effort to further define therapeutic options.

\section{Conclusion}

The CSC hypothesis appears to have a promising role in CRC tumor biology, despite the remaining unanswered questions related to this novel premise. By focusing on the identification and treatment of tumor progenitor cells, we may ultimately be able to improve screening, early detection, treatment, and prognostication ${ }^{55}$. Further identification of novel cell surface or cytoplasmic markers associated with CRCSC, and validation of known CRCSC could be useful in identifying tumors with poor prognosis. This approach could further enhance our ability to assess response to therapy and optimize treatment selection and intensity of post-treatment surveillance and follow-up. In order to translate these findings into clinical practice, prospective comprehensive analysis of a panel of CRCSC expression in large groups of patients is imperative ${ }^{59}$. Moreover, there remains a paucity of data evaluating the prognostic significance of the co-expression of multiple CRCSC within the same tumor, nodal and distant metastases within the same patients ${ }^{14,59}$. Overall, the CSC hypothesis may herald a paradigm shift in oncologic diagnosis and treatment following further prospective evaluation.

\section{Contributing Author Declaration}

We certify that all individuals who qualify as authors have been listed; each has participated in one or more of the following areas: conception and design of this work, the acquisition and/or analysis of data, the writing, and/or critical revision of the document, and supervision of this cooperative research effort. All contributing authors approve of the submission of this version of the manuscript and assert that the document represents valid work. If information derived from another source was used in this manuscript, we obtained all necessary approvals to use it and made appropriate acknowledgements in the document. All contributing authors take public responsibility for this work.

\section{Disclaimer}

The views expressed in this manuscript are those of the authors and do not reflect the official policy of the Department of the Army, the Department of Defense or the United States Government.

\section{Copyright protection}

One of the contributing authors are military service members (or employees of the U.S. Government: AS), and this work was prepared as part of their official duties. Title 17 U.S.C. 105 provides the "Copyright protection under this title is not available for any work of the United States Government." Title 17 U.S.C. 101 defines a U.S. Government work as a work prepared by a military service member or employee of the U.S. Government as part of that person's official duties.

\section{Competing Interests}

The authors have declared that no competing interest exists.

\section{References}

1. Siegel R, Ward E, Brawley O, Jemal A. Cancer statistics, 2011: the impact of eliminating socioeconomic and racial disparities on premature cancer deaths. CA: a cancer journal for clinicians 2011;61:212-36.

2. Bilchik AJ, DiNome M, Saha S, et al. Prospective multicenter trial of staging adequacy in colon cancer: preliminary results. Arch Surg 2006;141:527-33.

3. Manfredi S, Bouvier AM, Lepage C, Hatem C, Dancourt V, Faivre J. Incidence and patterns of recurrence after resection for cure of colonic cancer in a well defined population. The British journal of surgery 2006;93:1115-22.

4. Galizia G, Gemei M, Del Vecchio L, et al. Combined CD133/CD44 expression as a prognostic indicator of disease-free survival in patients with colorectal cancer. Arch Surg 2012;147:18-24.

5. Falcone A, Ricci S, Brunetti I, et al. Phase III trial of infusional fluorouracil, leucovorin, oxaliplatin, and irinotecan (FOLFOXIRI) compared with infusional fluorouracil, leucovorin, and irinotecan (FOLFIRI) as first-line treatment for metastatic colorectal cancer: the Gruppo Oncologico Nord Ovest. Journal of clinical oncology : official journal of the American Society of Clinical Oncology 2007;25:1670-6.

6. Wolpin BM, Meyerhardt JA, Mamon HJ, Mayer RJ. Adjuvant treatment of colorectal cancer. CA: a cancer journal for clinicians 2007;57:168-85.

7. Pilati P, Mocellin S, Bertazza L, et al. Prognostic value of putative circulating cancer stem cells in patients undergoing hepatic resection for colorectal liver metastasis. Annals of surgical oncology 2012;19:402-8.

8. Fearon ER, Vogelstein B. A genetic model for colorectal tumorigenesis. Cell 1990;61:759-67.

9. Fan J, Peng Z, Zhou C, et al. Gene-expression profiling in Chinese patients with colon cancer by coupling experimental and bioinformatic genomewide gene-expression analyses: identification and validation of 
IFITM3 as a biomarker of early colon carcinogenesis. Cancer 2008;113:266-75.

10. Muller AD, Sonnenberg A. Protection by endoscopy against death from colorectal cancer. A case-control study among veterans. Archives of internal medicine 1995;155:1741-8.

11. van Rossum LG, van Rijn AF, Laheij RJ, et al. Random comparison of guaiac and immunochemical fecal occult blood tests for colorectal cancer in a screening population. Gastroenterology 2008;135:82-90.

12. Winawer SJ, Stewart ET, Zauber AG, et al. A comparison of colonoscopy and double-contrast barium enema for surveillance after polypectomy. National Polyp Study Work Group. The New England journal of medicine 2000;342:1766-72.

13. Stoop EM, de Haan MC, de Wijkerslooth TR, et al. Participation and yield of colonoscopy versus non-cathartic CT colonography in population-based screening for colorectal cancer: a randomised controlled trial. The lancet oncology 2012;13:55-64.

14. Rosman AS, Korsten MA. Meta-analysis comparing CT colonography, air contrast barium enema, and colonoscopy. The American journal of medicine 2007;120:203-10.

15. Sacher-Huvelin S, Coron E, Gaudric M, et al. Colon capsule endoscopy vs. colonoscopy in patients at average or increased risk of colorectal cancer. Alimentary pharmacology \& therapeutics 2010;32:1145-53.

16. Sidransky D, Tokino T, Hamilton SR, et al. Identification of ras oncogene mutations in the stool of patients with curable colorectal tumors. Science 1992;256:102-5.

17. Dong SM, Traverso G, Johnson C, et al. Detecting colorectal cancer in stool with the use of multiple genetic targets. Journal of the National Cancer Institute 2001:93:858-65.

18. Imperiale TF, Ransohoff DF, Itzkowitz SH, Turnbull BA, Ross ME. Fecal DNA versus fecal occult blood for colorectal-cancer screening in an average-risk population. The New England journal of medicine 2004;351:2704-14.

19. Adler A, Wegscheider K, Lieberman D, et al. Factors determining the quality of screening colonoscopy: a prospective study on adenoma detection rates, from 12134 examinations (Berlin colonoscopy project 3, BECOP-3). Gut 2012.

20. CDC. Cancer screening - United States, 2010. MMWR Morbidity and mortality weekly report 2012;61:41-5.

21. Shires DA, Divine G, Schum M, et al. Colorectal cancer screening use among insured primary care patients. The American journal of managed care 2011;17:480-8.

22. Holmes-Rovner M, Williams GA, Hoppough S, Quillan L, Butler R, Given CW. Colorectal cancer screening barriers in persons with low income. Cancer practice 2002;10:240-7.

23. Locker GY, Hamilton S, Harris J, et al. ASCO 2006 update of recommendations for the use of tumor markers in gastrointestinal cancer. Journal of clinical oncology : official journal of the American Society of Clinical Oncology 2006;24:5313-27.

24. Filella X, Molina R, Grau JJ, et al. Prognostic value of CA 19.9 levels in colorectal cancer. Annals of surgery 1992;216:55-9.

25. Blake KE, Dalbow MH, Concannon JP, et al. Clinical significance of the preoperative plasma carcinoembryonic antigen (CEA) level in patients with carcinoma of the large bowel. Diseases of the colon and rectum 1982;25:24-32.

26. Hundt S, Haug U, Brenner H. Blood markers for early detection of colorectal cancer: a systematic review. Cancer epidemiology, biomarkers \& prevention : a publication of the American Association for Cancer Research, cosponsored by the American Society of Preventive Oncology 2007;16:1935-53.

27. Kopreski MS, Benko FA, Borys DJ, Khan A, McGarrity TJ, Gocke CD. Somatic mutation screening: identification of individuals harboring K-ras mutations with the use of plasma DNA. Journal of the National Cancer Institute 2000;92:918-23.

28. De Gruttola VG, Clax P, DeMets DL, et al. Considerations in the evaluation of surrogate endpoints in clinical trials. summary of a National Institutes of Health workshop. Controlled clinical trials 2001;22:485-502.

29. Hartwell L, Mankoff D, Paulovich A, Ramsey S, Swisher E. Cancer biomarkers: a systems approach. Nature biotechnology 2006;24:905-8.

30. Khambata-Ford S, Garrett CR, Meropol NJ, et al. Expression of epiregulin and amphiregulin and K-ras mutation status predict disease control in metastatic colorectal cancer patients treated with cetuximab. Journal of clinical oncology : official journal of the American Society of Clinical Oncology 2007;25:3230-7.

31. Srivastava S, Gopal-Srivastava R. Biomarkers in cancer screening: a public health perspective. The Journal of nutrition 2002;132:2471S-5S.
32. Srivastava S, Henson DJ, Gazdar A. Molecular Pathology of Early Cancer. Amsterdam: IOS. 1998.

33. Ahlquist DA, Skoletsky JE, Boynton KA, et al. Colorectal cancer screening by detection of altered human DNA in stool: feasibility of a multitarget assay panel. Gastroenterology 2000;119:1219-27.

34. Madeleine MM, Brumback B, Cushing-Haugen KL, et al. Human leukocyte antigen class II and cervical cancer risk: a population-based study. The Journal of infectious diseases 2002;186:1565-74.

35. Tejpar S, Bertagnolli M, Bosman F, et al. Prognostic and predictive biomarkers in resected colon cancer: current status and future perspectives for integrating genomics into biomarker discovery. The oncologist 2010;15:390-404.

36. Van Cutsem E, Köhne C-H, Hitre E, et al. Cetuximab and chemotherapy as initial treatment for metastatic colorectal cancer. The New England journal of medicine 2009;360:1408-17.

37. Van Cutsem E, Köhne C-H, Láng I, et al. Cetuximab Plus Irinotecan, Fluorouracil, and Leucovorin As First-Line Treatment for Metastatic Colorectal Cancer: Updated Analysis of Overall Survival According to Tumor KRAS and BRAF Mutation Status. Journal of Clinical Oncology 2011;:1-10.

38. De Roock W, Claes B, Bernasconi D, et al. Effects of KRAS, BRAF, NRAS, and PIK3CA mutations on the efficacy of cetuximab plus chemotherapy in chemotherapy-refractory metastatic colorectal cancer: a retrospective consortium analysis. The lancet oncology 2010;11:753-62.

39. Lièvre $A$, Bachet J-B, Boige $V$, et al. KRAS mutations as an independent prognostic factor in patients with advanced colorectal cancer treated with cetuximab. Journal of Clinical Oncology 2008;26:374-9.

40. Prahallad A, Sun C, Huang S, et al. Unresponsiveness of colon cancer to $\mathrm{BRAF}(\mathrm{V} 600 \mathrm{E})$ inhibition through feedback activation of EGFR. Nature 2012;483:100-3.

41. Thibodeau SN, Bren G, Schaid D. Microsatellite instability in cancer of the proximal colon. Science (New York, NY) 1993;260:816-9.

42. Tejpar S, Bertagnolli M, Bosman F, et al. Prognostic and predictive biomarkers in resected colon cancer: current status and future perspectives for integrating genomics into biomarker discovery. The oncologist 2010;15:390-404.

43. Gryfe R, Kim H, Hsieh ET, et al. Tumor microsatellite instability and clinical outcome in young patients with colorectal cancer. The New England journal of medicine 2000;342:69-77.

44. Ribic CM, Sargent DJ, Moore MJ, et al. Tumor microsatellite-instability status as a predictor of benefit from fluorouracil-based adjuvant chemotherapy for colon cancer. The New England journal of medicine 2003;349:247-57.

45. Sinicrope FA, Sargent DJ. Clinical implications of microsatellite instability in sporadic colon cancers. Current opinion in oncology 2009;21:369-73.

46. O'Brien CA, Pollett A, Gallinger S, Dick JE. A human colon cancer cell capable of initiating tumour growth in immunodeficient mice. Nature 2007;445:106-10.

47. Ricci-Vitiani L, Lombardi DG, Pilozzi E, et al. Identification and expansion of human colon-cancer-initiating cells. Nature 2007;445:111-5.

48. Visvader JE, Lindeman GJ. Cancer stem cells in solid tumours: accumulating evidence and unresolved questions. Nature reviews Cancer 2008;8:755-68

49. Xin HW, Hari DM, Mullinax JE, et al. Tumor-initiating label-retaining cancer cells in human gastrointestinal cancers undergo asymmetric cell division. Stem Cells 2012;30:591-8.

50. Avital I, Moreira AL, Klimstra DS, et al. Donor-derived human bone marrow cells contribute to solid organ cancers developing after bone marrow transplantation. Stem Cells 2007;25:2903-9.

51. Hari D, Xin HW, Jaiswal K, et al. Isolation of live label-retaining cells and cells undergoing asymmetric cell division via nonrandom chromosomal cosegregation from human cancers. Stem cells and development 2011;20:1649-58.

52. Cohnheim. Congenitales, quergestreiftes Muskelsarcom der Niere. Virchows Archiv fur Pathologische and Physiologic and fur Klinische Medizin 1875;65:64-9.

53. Nowell PC. The clonal evolution of tumor cell populations. Science 1976;194:23-8.

54. Hsiao YH, Chou MC, Fowler C, Mason JT, Man YG. Breast cancer heterogeneity: mechanisms, proofs, and implications. Journal of Cancer 2010;1:6-13.

55. Papailiou J, Bramis KJ, Gazouli M, Theodoropoulos G. Stem cells in colon cancer. A new era in cancer theory begins. International journal of colorectal disease 2011;26:1-11.

56. Vaiopoulos AG, Kostakis ID, Koutsilieris M, Papavassiliou AG. Colorectal cancer stem cells. Stem Cells 2012;30:363-71. 
57. Subramaniam D, Ramalingam S, Houchen CW, Anant S. Cancer stem cells: a novel paradigm for cancer prevention and treatment. Mini reviews in medicinal chemistry 2010;10:359-71.

58. Marquardt JU, Thorgeirsson SS. Stem cells in hepatocarcinogenesis: evidence from genomic data. Seminars in liver disease 2010;30:26-34.

59. Lugli A, Iezzi G, Hostettler I, et al. Prognostic impact of the expression of putative cancer stem cell markers CD133, CD166, CD44s, EpCAM, and ALDH1 in colorectal cancer. British journal of cancer 2010;103:382-90.

60. Willis ND, Przyborski SA, Hutchison CJ, Wilson RG. Colonic and colorectal cancer stem cells: progress in the search for putative biomarkers. Journal of anatomy 2008;213:59-65.

61. Deng S, Yang X, Lassus H, et al. Distinct expression levels and patterns of stem cell marker, aldehyde dehydrogenase isoform 1 (ALDH1), in human epithelial cancers. PloS one 2010;5:e10277.

62. Dalerba P, Dylla SJ, Park IK, et al. Phenotypic characterization of human colorectal cancer stem cells. Proceedings of the National Academy of Sciences of the United States of America 2007;104:10158-63.

63. Kemper K, Grandela C, Medema JP. Molecular identification and targeting of colorectal cancer stem cells. Oncotarget 2010;1:387-95.

64. Zou J, Yu XF, Bao ZJ, Dong J. Proteome of human colon cancer stem cells: a comparative analysis. World journal of gastroenterology : WJG 2011;17:1276-85.

65. Langan RC, Mullinax JE, Ray S, et al. A Pilot Study Assessing the Potential Role of non-CD133 Colorectal Cancer Stem Cells as Biomarkers. Journal of Cancer. 2012; 3:231-240. doi:10.7150/jca.4542.

66. Mulder JW, Kruyt PM, Sewnath M, et al. Colorectal cancer prognosis and expression of exon-v6-containing CD44 proteins. Lancet 1994;344:1470-2.

67. Wielenga VI, van der Voort R, Mulder JW, et al. CD44 splice variants as prognostic markers in colorectal cancer. Scandinavian journal of gastroenterology 1998;33:82-7.

68. Coppola D, Hyacinthe M, Fu L, et al. CD44V6 expression in human colorectal carcinoma. Human pathology 1998;29:627-35.

69. Patriarca C, Macchi RM, Marschner AK, Mellstedt H. Epithelial cell adhesion molecule expression (CD326) in cancer: a short review. Cancer treatment reviews 2012;38:68-75.

70. Gastl G, Spizzo G, Obrist P, Dunser M, Mikuz G. Ep-CAM overexpression in breast cancer as a predictor of survival. Lancet 2000;356:1981-2.

71. Prince S, Zeidman A, Dekel Y, Ram E, Koren R. Expression of epithelial cell adhesion molecule in gallbladder carcinoma and its correlation with clinicopathologic variables. American journal of clinical pathology 2008;129:424-9.

72. Gosens MJ, van Kempen LC, van de Velde CJ, van Krieken JH, Nagtegaal ID. Loss of membranous Ep-CAM in budding colorectal carcinoma cells. Modern pathology : an official journal of the United States and Canadian Academy of Pathology, Inc 2007;20:221-32.

73. Patel BB, Yu Y, Du J, Levi E, Phillip PA, Majumdar AP. Age-related increase in colorectal cancer stem cells in macroscopically normal mucosa of patients with adenomas: a risk factor for colon cancer. Biochemical and biophysical research communications 2009;378:344-7.

74. Weichert W, Knosel T, Bellach J, Dietel M, Kristiansen G. ALCAM/CD166 is overexpressed in colorectal carcinoma and correlates with shortened patient survival. Journal of clinical pathology 2004;57:1160-4.

75. Choi D, Lee HW, Hur KY, et al. Cancer stem cell markers CD133 and CD24 correlate with invasiveness and differentiation in colorectal adenocarcinoma. World journal of gastroenterology : WJG 2009;15:2258-64.

76. Lim SC, Oh SH. The role of CD24 in various human epithelial neoplasias. Pathology, research and practice 2005;201:479-86.

77. Weichert W, Denkert C, Burkhardt M, et al. Cytoplasmic CD24 expression in colorectal cancer independently correlates with shortened patient survival. Clinical cancer research : an official journal of the American Association for Cancer Research 2005;11:6574-81.

78. Barker N, van Es JH, Kuipers J, et al. Identification of stem cells in small intestine and colon by marker gene Lgr5. Nature 2007;449:1003-7.

79. Walker F, Zhang H-H, Odorizzi A, Burgess AW. LGR5 Is a Negative Regulator of Tumourigenicity, Antagonizes Wnt Signalling and Regulates Cell Adhesion in Colorectal Cancer Cell Lines. PloS one 2011;6:e22733.

80. Merlos-Suarez A, Barriga FM, Jung P, et al. The intestinal stem cell signature identifies colorectal cancer stem cells and predicts disease relapse. Cell stem cell 2011;8:511-24.

81. Takahashi H, Ishii H, Nishida N, et al. Significance of Lgr5(+ve) cancer stem cells in the colon and rectum. Annals of surgical oncology 2011;18:1166-74. 